\title{
A high LDL-C to HDL-C ratio predicts poor prognosis for initially metastatic colorectal cancer patients with elevations in LDL-C
}

This article was published in the following Dove Press journal:

OncoTargets and Therapy

27 October 2015

Number of times this article has been viewed

Fangxin Liao, ${ }^{1,2, *}$ Wenzhuo $\mathrm{He},{ }^{1,2, *}$ Chang Jiang, ${ }^{1,2}$ Chenxi Yin, ${ }^{1,2}$ Guifang Guo, ${ }^{1,2}$ Xuxian Chen, ${ }^{1,2}$ Huijuan Qiu, ${ }^{1,2}$ Yuming Rong, ${ }^{1,2}$ Bei Zhang, ${ }^{1,2}$ Dazhi $\mathrm{Xu},{ }^{1,3}$ Liangping $\mathrm{Xia}^{1,2}$

'State Key Laboratory of Oncology in South China, Collaborative Innovation Center for Cancer Medicine, ${ }^{2}$ VIP Region, ${ }^{3}$ Gastric and Pancreatic Surgery, Sun Yatsen University Cancer Center, Guangzhou, People's Republic of China

*These authors contributed equally to this work
Correspondence: Liangping Xia

VIP Region, Sun Yat-sen University Cancer Center, Guangzhou 510060, People's Republic of China

Tel +86208734 3107

Fax +86 2087343392

Email xialiangping@।63.com

Dazhi Xu

Gastric and Pancreatic Surgery, Sun Yat-sen University Cancer Center, Guangzhou 510060,

People's Republic of China

Tel +86 2087343737

Fax +862087343392

Email xudzh@sysucc.org.cn
Abstract: Although lipid disequilibrium has been documented for several types of cancer including colorectal cancer (CRC), it remains unknown whether lipid parameters are associated with the outcome of metastatic $\mathrm{CRC}(\mathrm{mCRC})$ patients. Here, we retrospectively examined the lipid profiles of $453 \mathrm{mCRC}$ patients and investigated whether any of the lipid parameters correlated with the outcome of mCRC patients. Pretreatment serum lipids, including triglyceride, cholesterol, high-density lipoprotein cholesterol (HDL-C), and low-density lipoprotein cholesterol (LDL-C), were collected in 453 initially mCRC patients. The LDL-C to HDL-C ratio (LHR) was calculated and divided into the first, second, and third tertiles. Univariate and multivariate analyses were performed to evaluate the impact of lipids on overall survival (OS) and progression-free survival (PFS). Nearly two-fifths of the patients (41.3\%) exhibited elevations in LDL-C while most patients $(88.3 \%)$ showed normal HDL-C levels. Decreased HDL-C $(P=0.542)$ and increased LDL-C $(P=0.023)$ were prognostic factors for poor OS, while triglyceride $(P=0.542)$ and cholesterol $(P=0.215)$ were not. Multivariate analysis revealed that LDL-C ( $P=0.031)$ was an independent prognostic factor. Triglyceride, cholesterol, HDL-C, and LDL-C did not correlate with PFS. Among patients with elevations in LDL-C levels, patients in the third tertile of the LHR had a markedly shorter median OS compared to those in the first or second tertile $(P=0.012)$. Thus, increased LDL-C level is an independent prognostic factor for poor prognosis in $\mathrm{mCRC}$ patients, and a high LHR predicts poor prognosis for initially $\mathrm{mCRC}$ patients with elevations in LDL-C.

Keywords: high low-density lipoprotein cholesterol, high-density lipoprotein cholesterol, metastatic colorectal cancer

\section{Introduction}

Colorectal cancer (CRC) is one of the most prevalent malignancies globally. While local $\mathrm{CRC}$ has a more favorable outcome with a 5-year survival rate of $90 \%$, the presence of distant metastasis adversely impacts the survival of CRC patients with a 5 -year survival of merely $12 \%{ }^{1}$ despite the best currently available treatment modalities. Prevention, early diagnosis, and treatment can greatly reduce the incidence and mortality of metastatic CRC (mCRC) patients. It is also important to identify prognostic markers for $\mathrm{mCRC}$ for risk mitigation.

Cancer patients are considered to be in a constant state of malnutrition, ${ }^{2}$ likely due to hypermetabolism and cachexia. Lipid disequilibrium has been well documented in cancer patients. ${ }^{3}$ Saito et $\mathrm{al}^{4}$ followed up a total of 16,217 liver cancer patients over 25 years and found that low low-density lipoprotein cholesterol (LDL-C) levels were associated with elevated risk of liver cancer mortality and that this may be a predictive 
marker for liver-cancer-related death. In a prospective study of 244 breast cancer patients, Rodrigues et $\mathrm{al}^{5}$ found that the disease-free survival of breast cancer patients within the third LDL-C tertile were $12 \%$ higher than that of patients in the first tertile. It also has been found that CRC patients with distant metastases had markedly higher levels of total cholesterol (TC), LDL-C, and LDL-C to high-density lipoprotein cholesterol (HDL-C) ratio compared with patients without metastases, and the presence of metastases was positively associated with TC, LDL-C, and the LDL-C/ HDL-C ratio (LHR). ${ }^{6}$ Liu et al $^{7}$ retrospectively examined the fasting lipid profile of 968 patients undergoing curative resection for primary CRC and found that both LDL-C levels and LHR were independently associated with advanced N2 stage in male CRC patients and that the LHR could be a more effective biomarker for N2 stage CRC than LDL-C levels alone.

Although lipid disequilibrium has been documented for several types of cancer including CRC, ${ }^{3,8}$ it remains unknown whether lipid parameters are associated with the outcome of mCRC patients. In this study, we retrospectively examined the lipid profiles of $453 \mathrm{mCRC}$ patients at a single tertiary care institution in the People's Republic of China and investigated whether any of the lipid parameters correlated with the outcome of mCRC patients.

\section{Patients and methods Patients}

Prior to use of the patients' sera, written informed consent was obtained from each of the participants, and the experiment was approved by the Institute Research Ethics Committee of Cancer Center of Sun Yat-sen University, Guangzhou, People's Republic of China. We retrospectively collected the clinicopathologic data of CRC patients who received medical treatment between January 1, 2005 and December 31, 2010 at Sun Yat-sen University Cancer Center. A patient was eligible for inclusion in the study: 1) if the patient had pathologically proven TNM stage IV CRC; 2) if the patient had completed at least three cycles of chemotherapy, including oxaliplatin- or irinotecanbased regimen; 3 ) if the patient had an Eastern Cooperative Oncology Group status score of 2 or less. A patient was excluded from the study: 1) if the patient had records of blood biochemical test before treatment; 2) if the patient did not have follow-up data; 3 ) if the patient had two or more primary tumors. The study protocol was approved by the local institutional review board at the authors' affiliated institution.

\section{Biochemical determinations}

Baseline serum triglyceride, cholesterol, LDL-C, and HDL-C were routinely determined using a Hitachi Automatic Analyzer 7600-020 (Hitachi, Tokyo, Japan) when patients first presented at the hospital. The LHR was calculated and divided into the first, second, and third tertiles.

\section{Patient follow-up}

Patients were followed up by telephone interview twice a year to learn whether they were alive or not. The primary endpoint of this study was overall survival (OS), defined as the time interval from diagnosis to death of any cause or the time of the last follow-up visit. The secondary endpoint was progression-free survival (PFS) of first-line chemotherapy, which was defined as the time interval from the first administration of chemotherapy to the date of disease progression or death.

\section{Statistical analysis}

Descriptive statistics were used to describe patient demographic and baseline characteristics. Normally distributed data were expressed as median \pm standard deviation and analyzed using the SPSS version 13.0 (SPSS Inc., Chicago, IL, USA). Nonnormally distributed data were expressed as median. Kaplan-Meier method was used to calculate survival curves, and log-rank test was used to compare differences. We performed multivariate analysis using Cox proportional hazards model, and independent significance was tested by backward elimination of insignificant explanatory variables. A $P$-value $\leq 0.05$ was considered as significant.

\section{Results}

\section{Patient demographic and baseline characteristics}

The study flowchart is shown in Figure 1. Five hundred sixty-six CRC patients were identified in the database during the review period. Sixty-eight patients failed to meet the eligibility criteria and were excluded from the study. Among them, three patients declined treatment; 29 patients underwent palliative primary tumor resection but received no chemotherapy; and 36 patients only completed one or two cycles of chemotherapy. Consequently, a total of 453 patients were included in the final analysis. The demographic and baseline characteristics of these patients are shown in Table 1. Their median age was 59 (range, 18-89) years, and the majority of these patients were aged at least 50 years $(62.9 \%)$ and were male $(65.3 \%)$. CRC was located in the 
Patients initially diagnosed as metastatic colorectal cancer patients between January 2005 and December 2010 at Sun Yat-sen University

Cancer Center $(n=566)$

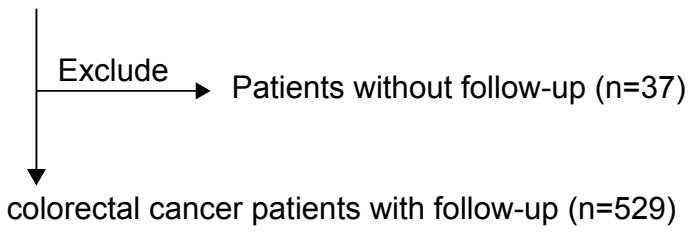

Metastatic colorectal cancer patients with follow-up $(n=529)$

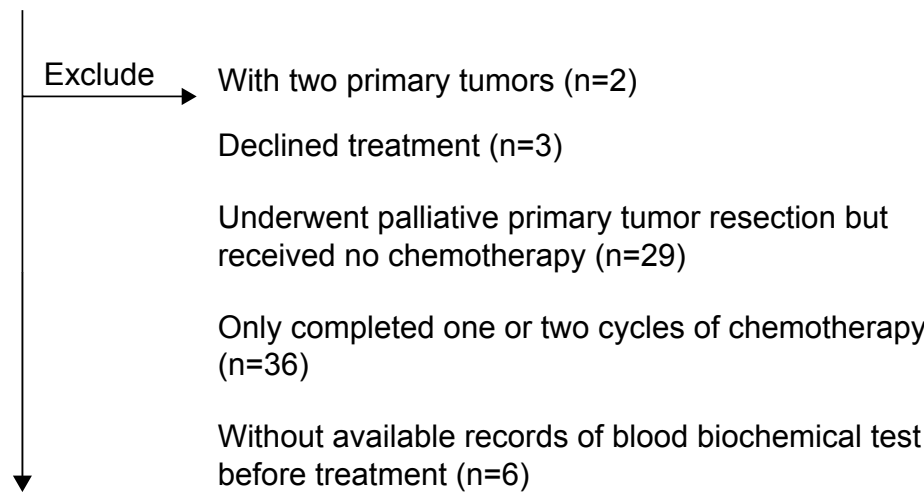

453 patients were included in the final analysis

Figure I The study flowchart.

Table I Demographic and baseline characteristics of patients with metastatic colorectal cancer

\begin{tabular}{|c|c|}
\hline Variables & $\mathbf{N}(\%)$ \\
\hline Number of patients & $453(100)$ \\
\hline \multicolumn{2}{|l|}{ Age (years) } \\
\hline$>50$ & $285(62.9)$ \\
\hline \multicolumn{2}{|l|}{ Sex } \\
\hline Male & $296(65.3)$ \\
\hline \multicolumn{2}{|l|}{ Location } \\
\hline Right & $176(38.9)$ \\
\hline Left & $146(32.2)$ \\
\hline Rectal & $|3|(28.9)$ \\
\hline \multicolumn{2}{|l|}{ Palliative primary tumor resection } \\
\hline Yes & $325(71.7)$ \\
\hline \multicolumn{2}{|l|}{ Chemotherapy } \\
\hline \multicolumn{2}{|l|}{ First-line } \\
\hline Oxaliplatin-based regimen & $303(66.9)$ \\
\hline Irinotecan-based regimen & $150(33.1)$ \\
\hline \multicolumn{2}{|l|}{ Second-line } \\
\hline Oxaliplatin-based regimen & $96(21.2)$ \\
\hline Irinotecan-based regimen & $219(48.3)$ \\
\hline None & $138(30.5)$ \\
\hline \multicolumn{2}{|l|}{ Number of metastatic organs } \\
\hline I & $275(60.7)$ \\
\hline 2 & $136(30.0)$ \\
\hline$\geq 3$ & $42(9.3)$ \\
\hline \multicolumn{2}{|l|}{ CEA (ng/mL) } \\
\hline$>5$ & $374(82.6)$ \\
\hline \multicolumn{2}{|l|}{ BMI $\left(\mathrm{kg} / \mathrm{m}^{2}\right)$} \\
\hline$>24$ & $105(23.2)$ \\
\hline$\leq 24$ & $348(76.8)$ \\
\hline
\end{tabular}

Abbreviations: CEA, carcinoembryonic antigen; BMl, body mass index. right colon in $38.9 \%$ of the patients followed by the left colon $(32.2 \%)$ and the rectum (28.9\%). Carcinoembryonic antigen was $5 \mathrm{ng} / \mathrm{mL}$ or above in most cases $(82.6 \%)$. The majority of the patients $(60.7 \%)$ had metastasis to one organ, $30.0 \%$ had metastasis to two organs, and 9.3\% had metastasis to at least three organs.

\section{Treatment characteristics of the patients}

The majority of patients $(71.7 \%)$ underwent palliative primary tumor resection (Table 1). The median number of chemotherapy cycles was 7 (range, 3-12). For first-line chemotherapy, two-thirds of the patients $(66.9 \%)$ received oxaliplatin-based regimen while the remaining one-third (33.1\%) received irinotecan-based regimen. For secondline chemotherapy, approximately half of the patients $(48.3 \%)$ received irinotecan-based regimen and one-fifth of the patients $(21.2 \%)$ received oxaliplatin-based regimen. Approximately one-third of the patients (30.5\%) received no second-line chemotherapy.

\section{The lipid profile of the patients}

The distribution of HDL-C, LDL-C, cholesterol, and triglycerides is shown in Table 2 . The majority of the patients $(78.2 \%)$ had normal triglyceride levels while nearly one-fifth of the patients $(21.9 \%)$ had increased serum triglyceride contents. Meanwhile, most patients $(89.2 \%)$ had normal cholesterol 
Table 2 The distributions of triglyceride, cholesterol, LDL-C, HDL-C, and LHR in patients with metastatic colorectal cancer

\begin{tabular}{lllllll}
\hline Variable & $\begin{array}{l}\text { Normal } \\
\text { referent value }\end{array}$ & Median & Mean \pm SD & $\begin{array}{l}\text { Patients with } \\
\text { elevated levels (n, \%) }\end{array}$ & $\begin{array}{l}\text { Patients with } \\
\text { normal levels (n, \%) }\end{array}$ & $\begin{array}{l}\text { Patients with } \\
\text { decreased levels (n, \%) }\end{array}$ \\
\hline Triglyceride (mmol/L) & $0.2-1.7$ & 1.25 & $1.35 \pm 0.39$ & $99(21.85)$ & $354(78.15)$ & $0(0)$ \\
Cholesterol (mmol/L) & $2.1-6.4$ & 4.96 & $5.15 \pm 1.18$ & $49(I 0.82)$ & $404(89.18)$ & $0(0)$ \\
LDL-C (mmol/L) & $2.2-3.4$ & 3.12 & $3.32 \pm 1.15$ & $187(41.28)$ & $216(47.68)$ & $50(11.04)$ \\
HDL-C (mmol/L) & $0.78-2.2$ & 1.12 & $1.14 \pm 0.32$ & $4(0.89)$ & $400(88.30)$ & $49(10.81)$ \\
ALT (U/L) & $0-40$ & 21.15 & $27.95 \pm 22.69$ & $61(13.47)$ & $392(86.53)$ & $0(0)$ \\
AST (U/L) & $0-45$ & 20.4 & $43.17 \pm 87.76$ & $91(20.09)$ & $362(79.91)$ & $0(0)$ \\
LHR & & 3.03 & $3.30 \pm 1.89$ & & & \\
\hline
\end{tabular}

Abbreviations: HDL-C, high-density lipoprotein cholesterol; LDL-C, low-density lipoprotein cholesterol; LHR, LDL-C to HDL-C ratio; SD, standard deviation; U/L, units per liter; ALT, alanine aminotransferase; AST, aspartate aminotransferase.

contents with one in ten patients $(10.8 \%)$ had increased cholesterol levels. Slightly less than half of the patients (47.7\%) had normal LDL-C while nearly two-fifths of the patients (41.3\%) exhibited elevations in LDL-C. Most patients $(88.3 \%)$ showed normal HDL-C levels, with about one in ten patients (10.8\%) exhibiting reduced HDL-C levels. The median LHR was 3.02 (range, 0.61-28.38). Approximately 151 (33.33\%) patients fell within the third tertile (3.51-28.38), $151(33.33 \%)$ patients fell within the second tertile (2.55-3.50), and 151 $(33.33 \%)$ patients fell within the first tertile $(0.61-2.55)$.

\section{OS and PFS}

The median duration of follow-up time was 20 (range, 2-86) months. The Kaplan-Meier survival curve is shown in Figure 2. The median OS was 19.60 (range, 1.87-70.30) months and the median PFS was 6.47 (range, 1.57-58.03) months. One hundred thirty-eight $(30.5 \%)$ patients were still alive at the last follow-up visit on September 30, 2014.

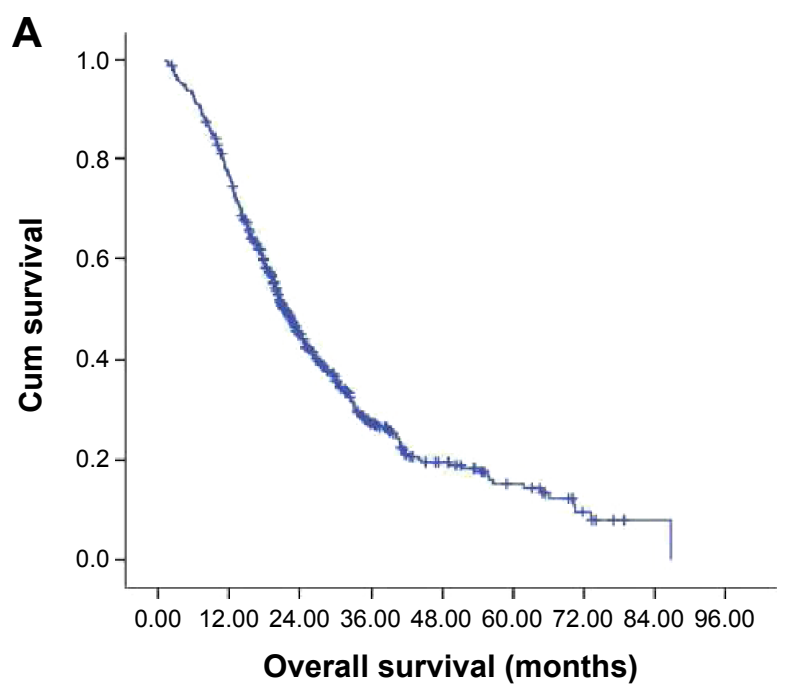

Figure 2 The Kaplan-Meier curve for OS (A) and PFS (B) of the study patients. Abbreviations: OS, overall survival; PFS, progression-free survival; Cum, cumulative.

\section{LDL-C was an independent prognostic determinant of OS in $\mathrm{MCRC}$ patients}

We were interested in and examined whether HDL-C, LDL-C, cholesterol, and triglyceride were prognostic determinants of mCRC patients. Our univariate analysis revealed that LDL-C $(P=0.023)$ and HDL-C $(P=0.035)$ were significant prognostic factors, while cholesterol $(P=0.215)$ and triglyceride $(P=0.542)$ were not. The median OS of patients with elevated LDL-C levels was 19.17 (range, 11.87-61.80) months while that of patients with normal LDL-C levels was 20.07 (range, 2.77-70.30) months (Figure $3 \mathrm{~A}$ and Table 3 ). The median OS of patients with decreased LDL-C levels was 18.63 (range, 1.87-47.30) months. HDL-C was also a prognostic factor ( $P=0.035)$ while cholesterol $(P=0.215)$ and triglycerides $(P=0.542)$ were not (Table 3$)$. The OS of patients with decreased and normal LDL-C was similar $(P=0.652)$. In contrast, the median OS of patients with elevated HDL-C
B

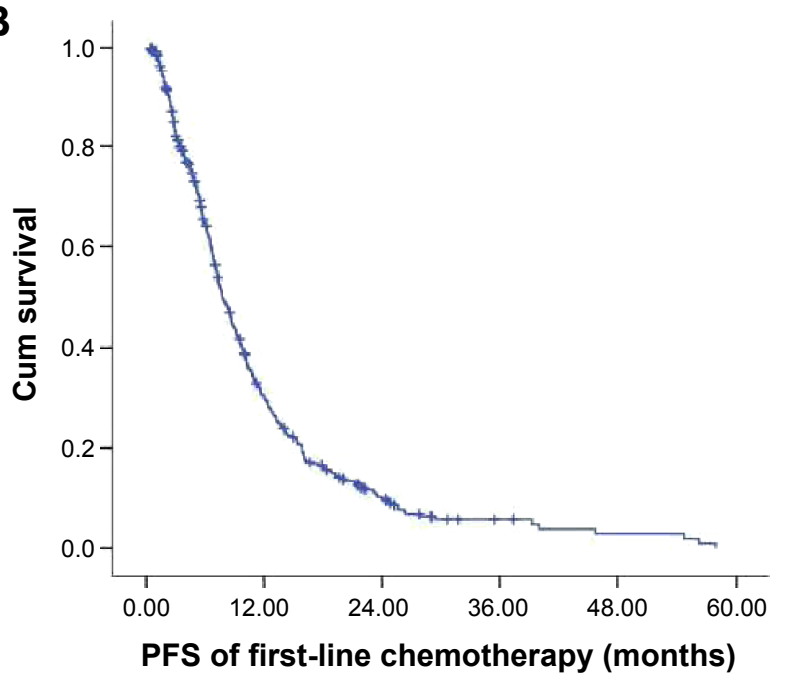



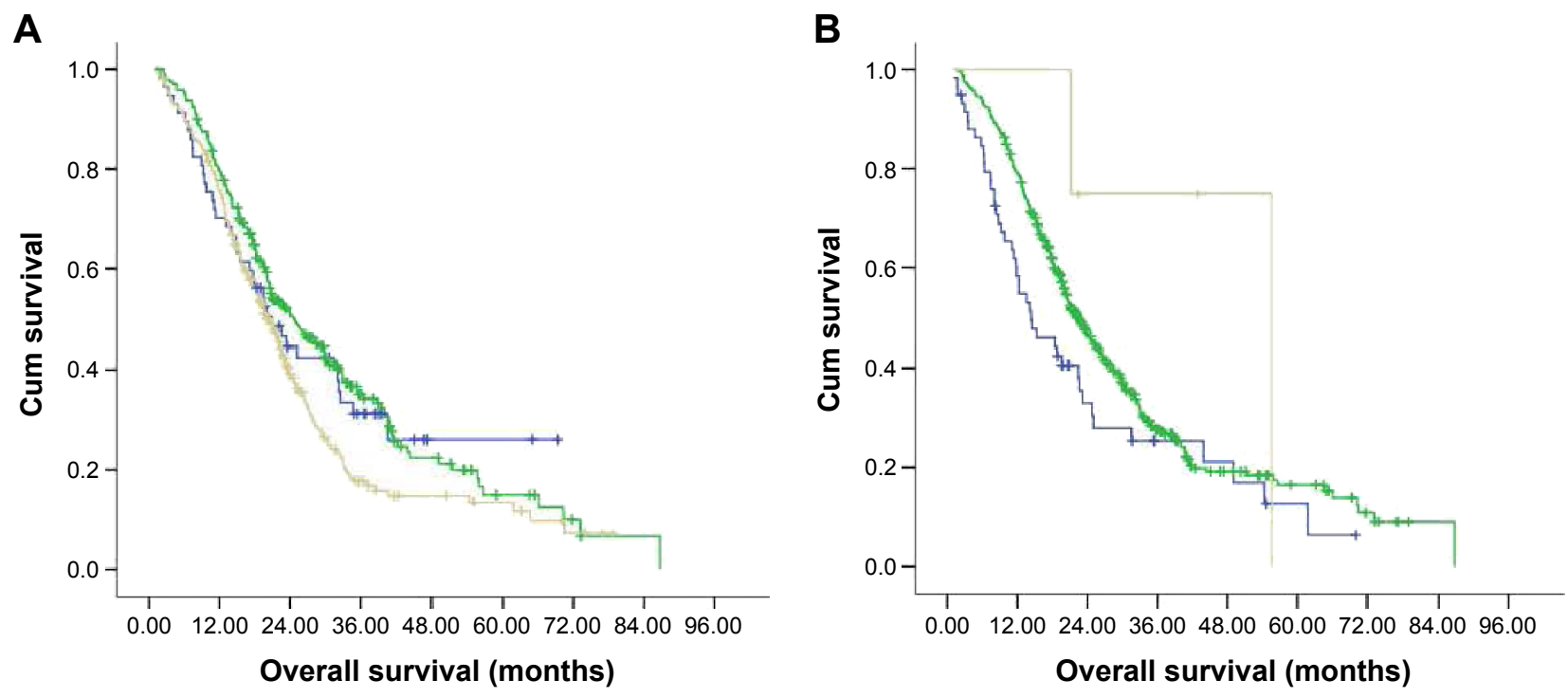

$\sqcap$ Decreased $\Pi$ Normal range $\Pi$ Increased

Figure 3 The Kaplan-Meier curve for OS of the study patients stratified by LDL-C (A) and HDL-C (B).

Abbreviations: OS, overall survival; Cum, cumulative; HDL-C, high-density lipoprotein cholesterol; LDL-C, low-density lipoprotein cholesterol.

and those with normal or decreased HDL-C was 22.43 (range, 21.20-42.93) months and 16.28 (range, 1.87-70.30) months, respectively $(P=0.006)$ (Figure 3B). Furthermore, multivariate analysis revealed that only LDL-C $(P=0.031)$ was an independent predictor of outcome while HDL-C $(P=0.337)$ was not (Table 4).

\section{HDL-C, LDL-C, cholesterol, or triglyceride shows no correlation with PFS}

We further evaluated whether HDL-C, LDL-C, cholesterol, and triglyceride correlated with PFS of mCRC patients (Table 3).
The median PFS of patients with elevated LDL-C levels was 8.16 (range, 3.28-45.26) months while that of patients with normal LDL-C levels was 7.28 (range, 2.03-58.03) months (Figure $4 \mathrm{~A}$ and Table 3 ). The median PFS of patients with decreased LDL-C levels was 5.56 (range, 1.57-24.40) months. The median PFS of patients with elevated HDL-C and those with normal or decreased HDL-C was 7.85 (range, 1.62-58.03) months and 5.52 (range, 1.57-45.26) months, respectively $(P=0.865)$ (Figure 4B). None of the parameters including HDL-C, LDL-C, cholesterol, and triglyceride correlated with PFS.

Table 3 The OS and PFS of patients with metastatic colorectal cancer according to levels of triglyceride, cholesterol, LDL-C, and HDL-C $(n=453)$

\begin{tabular}{|c|c|c|c|c|c|c|}
\hline Variable & Patients (n) & Number of events & $\begin{array}{l}\text { OS (months), } \\
\text { median and range }\end{array}$ & $P$-value & $\begin{array}{l}\text { PFS (months), } \\
\text { median and range }\end{array}$ & $P$-value \\
\hline Triglyceride & & & & 0.542 & & 0.984 \\
\hline Normal & 354 & 241 & 19.65 (I.87-70.30) & & $6.70(1.57-58.03)$ & \\
\hline Increased & 99 & 74 & $18.95(2.87-54.60)$ & & 9.34 (1.95-44.67) & \\
\hline Cholesterol & & & & 0.215 & & 0.127 \\
\hline Normal & 404 & 279 & 19.67 (2.70-70.30) & & 7.67 (2.03-58.03) & \\
\hline Increased & 49 & 36 & 14.70 (1.87-55.03) & & $6.46(1.57-47.54)$ & \\
\hline LDL-C & & & & 0.023 & & 0.162 \\
\hline Decreased & 50 & 32 & I8.63 (I.87-47.30) & & $5.56(1.57-24.40)$ & \\
\hline Normal & 216 & 152 & $20.07(2.77-70.30)$ & & $7.28(2.03-58.03)$ & \\
\hline Increased & 187 & $13 \mid$ & 19.17 (II.87-61.80) & & $8.16(3.28-45.26)$ & \\
\hline HDL-C & & & & 0.035 & & 0.865 \\
\hline Decreased & 49 & 35 & $12.27(1.87-61.80)$ & & $5.52(1.57-45.26)$ & \\
\hline Normal & 400 & 277 & 19.67 (I.87-70.30) & & $7.85(1.62-58.03)$ & \\
\hline Increased & 4 & 3 & $22.43(21.20-42.93)$ & & $6.35(2.42-28.74)$ & \\
\hline
\end{tabular}

Abbreviations: OS, overall survival; PFS, progression-free survival; HDL-C, high-density lipoprotein cholesterol; LDL-C, low-density lipoprotein cholesterol. 
Table 4 Multivariate analysis of LDL-C and HDL-C as prognostic determinants of OS patients with metastatic colorectal cancer

\begin{tabular}{lllll}
\hline Variable & B & P-value & $\operatorname{Exp}(B)$ & $\mathbf{9 5 \% ~ C l ~ f o r ~} \exp (B)$ \\
\hline LDL-C & -0.238 & 0.031 & 0.788 & $0.635-0.978$ \\
HDL-C & 0.683 & 0.337 & 1.980 & $0.491-7.987$
\end{tabular}

Abbreviations: OS, overall survival; HDL-C, high-density lipoprotein cholesterol; LDL-C, low-density lipoprotein cholesterol; $\mathrm{Cl}$, confidence interval.

\section{The LHR is a novel prognostic factor}

Our findings indicated that LDL-C negatively correlated with OS while HDL-C positively correlated with OS. We speculated that the LHR could be a more discriminative prognostic predictor. The median OS of $\mathrm{mCRC}$ patients in the second or third tertile of the LHR was 16.70 (range, 2.53-66.73) months, which was significantly shorter than that of patients in the first tertile of the LHR $(P<0.001)$ (median, 21.67 months; range, 1.87-70.30 months). To evaluate whether LHR was superior to LDL-C, we stratified patients according to their LDL-C levels. Among patients with elevations in LDL-C levels, patients in the third tertile of the LHR had a markedly shorter median OS (16.73 months; range, 11.87-60.47 months) compared to those in the first (22.77 months; range, 13.73-61.8 months) or second tertile (21.03 months; range, 12.67-61.0 months) $(P=0.012$; Figure 5 and Table 5). Although patients in the third tertile who had no elevations in LDL-C showed a lower median OS (15.62 months; range, 2.53-49.07 months), no statistically significant difference was observed in OS between these patients and patients in the first (21.17 months; range, 1.87-70.30 months) or second tertile (19.97 months; range, 2.50-66.73 months) of the LHR ( $P=0.073)$.

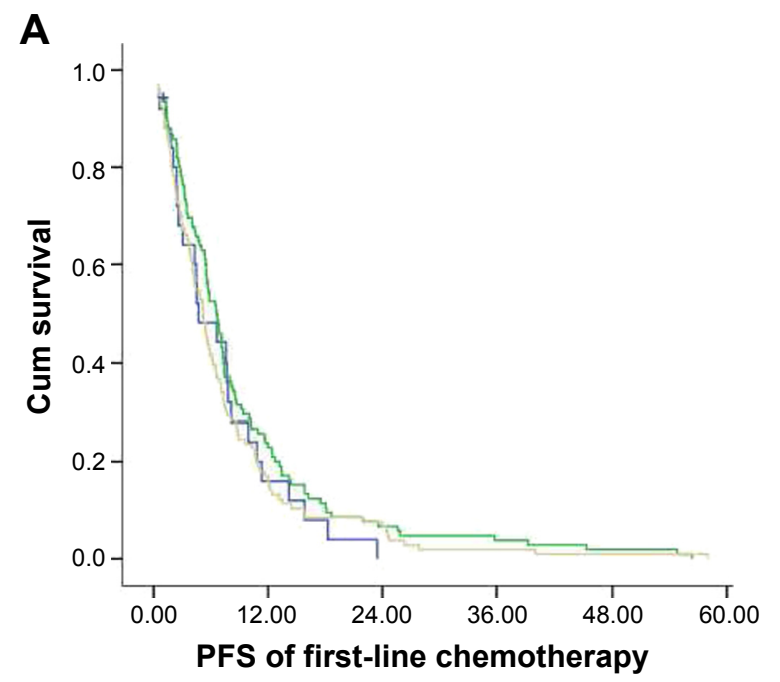

\section{Discussion}

Lipid disequilibrium has been documented for several types of cancer, including CRC. ${ }^{3,8}$ In this paper, we found that $41.28 \%$ of mCRC patients showed elevations in LDL-C. Our univariate analysis revealed that baseline LDL-C and HDL-C were prognostic determinants of $\mathrm{mCRC}$, and our multivariate analysis found that LDL-C was an independent prognostic predictor of mCRC. Furthermore, the LHR could also yield prognostic information, with a high LHR predicting an adverse outcome for patients with elevations in LDL-C.

Cholesterol is a structural component of the cell membrane and is localized in membrane microdomains that assemble the signal transduction machinery and associate to proteins implicated in key cellular signaling pathways that are closely associated with malignant transformation. ${ }^{9}$ LDL-C and HDL-C are lipoproteins responsible for cholesterol transportation. However, there has been scant knowledge available about LDL-C in cancer patients. This study was the first to examine the correlation between LDL-C, HDL-C, and the LHR and the OS of mCRC patients. Our findings are consistent with those reported by others. ${ }^{6}$ Notarnicola et $\mathrm{al}^{6}$ found that metastases in CRC patients were positively associated with LDL-C and the LHR. Liu et $\mathrm{al}^{7}$ also found that both LDL-C levels and the LHR were independently associated with advanced N2 stage in male CRC patients.

Although the relation between lipids and cancer has been known for years, most previous studies have focused on the influence of lipids on the incidence of tumor among healthy subjects. ${ }^{10,11}$ It was reported that people with low LDL-C level were more likely to develop a tumor. ${ }^{10,11}$

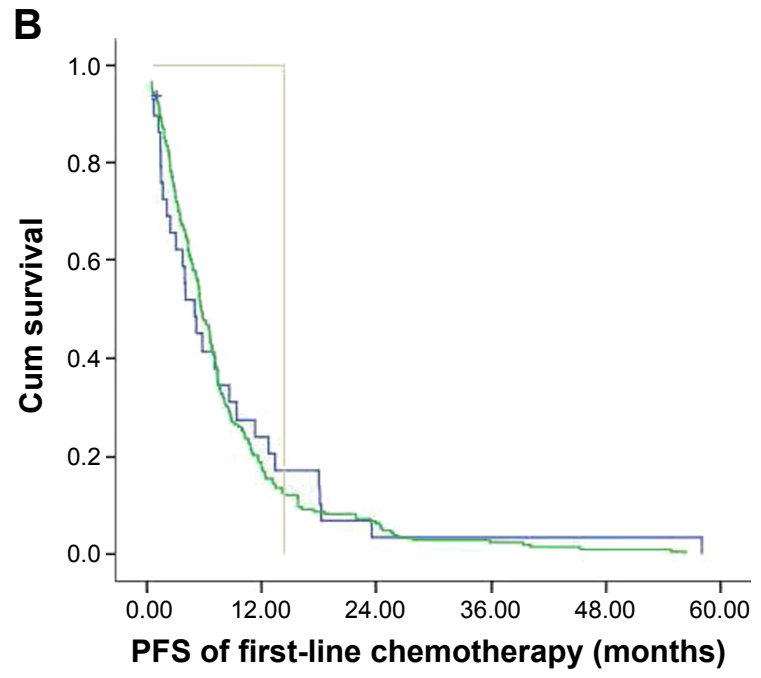

Figure 4 The Kaplan-Meier curve for PFS of the study patients stratified by LDL-C (A) and HDL-C (B).

Abbreviations: PFS, progression-free survival; Cum, cumulative; HDL-C, high-density lipoprotein cholesterol; LDL-C, low-density lipoprotein cholesterol. 


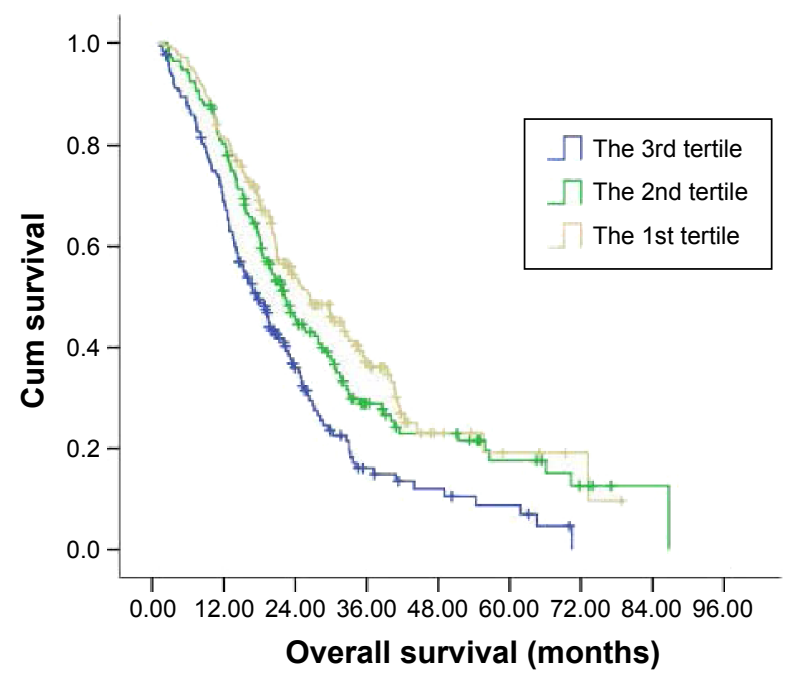

Figure 5 The Kaplan-Meier curve for OS of the study patients stratified by the LHR.

Abbreviations: OS, overall survival; Cum, cumulative; HDL-C, high-density lipoprotein cholesterol; LDL-C, low-density lipoprotein cholesterol; LHR, LDL-C to HDL-C ratio.

It was reported that most cancer patients had low LDL-C and cholesterol levels. ${ }^{8}$ Cancer patients are in poor nutritional status as a result of cachexia and poor appetite due to chemotherapy and other treatment modalities. Cancer cells could also take up and degrade a large amount of LDL-C in order to compose biomembrane, required for the division of malignant cells. ${ }^{12}$ Previous studies involved patients with various cancer types, including breast cancer, gastric cancer, and lung cancer. ${ }^{8}$ However, patients with different types of cancer might have different LDL-C profiles. For example, breast cancer patients had elevated LDL-C levels ${ }^{13}$ whereas gastric and lung cancer patients ${ }^{8}$ had decreased LDL-C levels. In this paper, we focused on mCRC patients, and

Table 5 The OS of patients with metastatic colorectal cancer according to the LHR

\begin{tabular}{lllll}
\hline & TI & T2 & T3 & P-value \\
\hline $\begin{array}{l}\text { Patients with } \\
\text { elevations in LDL-C }\end{array}$ & & & & 0.0 I \\
$\begin{array}{l}\text { Patients (n) } \\
\text { Number of events }\end{array}$ & 13 & 55 & 119 & \\
$\quad$ Median & 22.77 & 221.03 & 16.73 & \\
$\quad$ Range & $13.73-61.8$ & $12.67-61.0$ & $11.87-60.47$ & \\
Patients with no & & & & 0.07 \\
elevations in LDL-C & & & & \\
Patients (n) & 138 & 96 & 32 & \\
Number of events & 90 & 76 & 23 & \\
$\quad \begin{array}{l}\text { Median } \\
\text { Range }\end{array}$ & 21.17 & 19.97 & 15.62 & \\
\hline
\end{tabular}

Abbreviations: OS, overall survival; HDL-C, high-density lipoprotein cholesterol; LDL-C, low-density lipoprotein cholesterol; LHR, LDL-C to HDL-C ratio; TI, T2, and T3 represent the first, second, and third tertiles of LDL-C contents, respectively. found that $41.28 \%$ of these patients had increased LDL-C, which was an adverse prognostic factor for $\mathrm{mCRC}$ patients. We saw increased rather than decreased LDL-C levels in these patients which may be due to the fact that we focused on initially mCRC patients. Notarnicola et $\mathrm{al}^{6}$ reported that LDL-C levels may increase as CRC develops from the early stage to the advanced stage. Several other studies also support our result that increased LDL-C was a predictor for poor prognosis. Montel et $\mathrm{al}^{14}$ and Song et $\mathrm{a} \mathrm{l}^{15}$ reported that cancer cells expressed LDL-receptor-related protein, which could facilitate the growth and invasion of cancer cells. Another study reported ${ }^{16}$ that oxidized LDL could stimulate the proliferation of ovarian carcinoma cells. These results could help explain the unfavorable influence of LDL-C on OS observed in our study.

In our study, although both LDL-C and HDL-C were prognostic factors, only LDL-C was found to be independent. The exact reason was unknown. Tumor cells need plenty of lipids to maintain division. Both increased LDL-C and decreased HDL-C reflected that lipids were more likely to be transported into the blood rather than stored in the liver. We speculated that both increased LDL-C and decreased HDL-C indicated that tumor had resulted in altered lipid profile, in a way favoring the growth and division of tumor cells. The reason why only LDL-C was independent might be that tumor cells express LDL-C receptor and could directly uptake and degrade LDL-C. ${ }^{3}$ To fully consider the implications of both LDL-C and HDL-C, we also studied the prognostic value of the LHR, and found that the LHR could provide further information based on LDL-C. Thus, the LHR may be a promising prognostic factor in mCRC patients, especially those with elevations in LDL-C.

Since both preclinical and clinical data support that LDL-C plays a role in cancer patients, one important issue is whether bringing down LDL-C is of therapeutic value for mCRC patients. Although the impact of statin, a commonly used agent used to reduce lipid, on the incidence of cancer had been extensively studied, ${ }^{11,17-19}$ it was reported that statin was correlated with a significant reduction in CRC risk. ${ }^{20,21}$ To the best of our knowledge, no study had evaluated the therapeutic value of statin in mCRC patients. Further studies are urgently needed to evaluate the therapeutic effect of statins in mCRC patients.

There were several limitations in this study. First, the retrospective nature restricted its power. Second, it is not clear that whether the increased LDL-C was caused by CRC. In other words, there were two possible situations: 1) cancers which gained the ability to adjust lipid profile yield poor prognosis and 2) patients who had increased LDL-C yield 
poor prognosis when they developed CRC. Third, increased LDL-C itself was a risk factor. There was one possibility that there was no interaction between LDL-C and cancer, and the unfavorable prognosis was caused by risks resultant from increased LDL-C. Further prospective studies are expected to answer those questions.

In conclusion, we found that increased LDL-C level was a prognostic factor for poor prognosis in $\mathrm{mCRC}$ patients. Further prospective and multiple center studies are required, and the therapeutic value of statin in $\mathrm{mCRC}$ patients is also worth being explored.

\section{Acknowledgments}

The authors thank the patients and the colleagues at the Sun Yat-sen University Cancer Center who participated in this study. The abstract of this paper was presented at the 2014 World Congress as an oral presentation with interim findings. The abstract was published in Asia-Pacific Journal of Clinical Oncology titled as "A high LDL-C to HDL-C ratio predicts poor prognosis for initially metastatic colorectal cancer patients with elevations in LDL-C". This study was supported by grants from the Natural Science Foundation of Guangdong, China (2015A030313010), Science and Technology Program of Guangzhou, China (1563000305) and National Natural Science Foundation of China (81272641 and 81572409).

\section{Disclosure}

The authors report no conflicts of interest in this work.

\section{References}

1. Hu T, Yao Y, Yu S, et al. Clinicopathologic significance of CXCR4 and Nrf2 in colorectal cancer. J Biomed Res. 2013;27(4):283-290.

2. Rose G, Blackburn H, Keys A, et al. Colon cancer and blood-cholesterol. Lancet. 1974;1(7850):181-183.

3. Vitols S, Gahrton G, Bjorkholm M, Peterson C. Hypocholesterolaemia in malignancy due to elevated low-density-lipoprotein-receptor activity in tumour cells: evidence from studies in patients with leukaemia. Lancet. 1985;2(8465):1150-1154.

4. Saito N, Sairenchi T, Irie F, et al. Low serum LDL cholesterol levels are associated with elevated mortality from liver cancer in Japan: the Ibaraki Prefectural health study. Tohoku J Exp Med. 2013;229(3):203-211.
5. Rodrigues Dos Santos C, Fonseca I, Dias S, Mendes de Almeida JC. Plasma level of LDL-cholesterol at diagnosis is a predictor factor of breast tumor progression. BMC Cancer. 2014;14:132.

6. Notarnicola M, Altomare DF, Correale M, et al. Serum lipid profile in colorectal cancer patients with and without synchronous distant metastases. Oncology. 2005;68(4-6):371-374.

7. Liu YL, Qian HX, Qin L, Zhou XJ, Zhang B. Serum LDL-C and LDL-C/HDL-C ratio are positively correlated to lymph node stages in males with colorectal cancer. Hepatogastroenterology. 2011; 58(106):383-387.

8. Muntoni S, Atzori L, Mereu R, et al. Serum lipoproteins and cancer. Nutr Metab Cardiovasc Dis. 2009;19(3):218-225.

9. Lingwood D, Simons K. Lipid rafts as a membrane-organizing principle. Science. 2010;327(5961):46-50.

10. Jacobs D, Blackburn H, Higgins M, et al. Report of the conference on low blood cholesterol: mortality associations. Circulation. 1992;86(3): 1046-1060.

11. Emberson JR, Kearney PM, Blackwell L, et al. Lack of effect of lowering LDL cholesterol on cancer: meta-analysis of individual data from 175,000 people in 27 randomised trials of statin therapy. PLoS One. 2012; 7(1):e29849.

12. Mack JT, Townsend DM, Beljanski V, Tew KD. The ABCA2 transporter: intracellular roles in trafficking and metabolism of LDL-derived cholesterol and sterol-related compounds. Curr Drug Metab. 2007; 8(1):47-57.

13. Laisupasin P, Thompat W, Sukarayodhin S, Sornprom A, Sudjaroen Y. Comparison of serum lipid profiles between normal controls and breast cancer patients. $J$ Lab Physicians. 2013;5(1):38-41.

14. Montel V, Gaultier A, Lester RD, Campana WM, Gonias SL. The lowdensity lipoprotein receptor-related protein regulates cancer cell survival and metastasis development. Cancer Res. 2007;67(20):9817-9824.

15. Song H, Li Y, Lee J, Schwartz AL, Bu G. Low-density lipoprotein receptor-related protein 1 promotes cancer cell migration and invasion by inducing the expression of matrix metalloproteinases 2 and 9. Cancer Res. 2009;69(3):879-886.

16. Li AJ, Elmore RG, Chen IY, Karlan BY. Serum low-density lipoprotein levels correlate with survival in advanced stage epithelial ovarian cancers. Gynecol Oncol. 2010;116(1):78-81.

17. Kuoppala J, Lamminpaa A, Pukkala E. Statins and cancer: a systematic review and meta-analysis. Eur J Cancer. 2008;44(15):2122-2132.

18. Dale KM, Coleman CI, Henyan NN, Kluger J, White CM. Statins and cancer risk: a meta-analysis. JAMA. 2006;295(1):74-80.

19. Bonovas S, Filioussi K, Tsavaris N, Sitaras NM. Statins and cancer risk: a literature-based meta-analysis and meta-regression analysis of 35 randomized controlled trials. J Clin Oncol. 2006;24(30):4808-4817.

20. Poynter JN, Gruber SB, Higgins PD, et al. Statins and the risk of colorectal cancer. N Engl J Med. 2005;352(21):2184-2192.

21. Broughton T, Sington J, Beales IL. Statin use is associated with a reduced incidence of colorectal cancer: a colonoscopy-controlled casecontrol study. BMC Gastroenterol. 2012;12:36.
OncoTargets and Therapy

\section{Publish your work in this journal}

OncoTargets and Therapy is an international, peer-reviewed, open access journal focusing on the pathological basis of all cancers, potential targets for therapy and treatment protocols employed to improve the management of cancer patients. The journal also focuses on the impact of management programs and new therapeutic agents and protocols on
Dovepress

patient perspectives such as quality of life, adherence and satisfaction. The manuscript management system is completely online and includes a very quick and fair peer-review system, which is all easy to use. Visit http://www.dovepress.com/testimonials.php to read real quotes from published authors. 